\title{
Relationship between hepatic CTGF expression and routine blood tests at the time of liver transplantation for biliary atresia: hope or hype for a biomarker of hepatic fibrosis
}

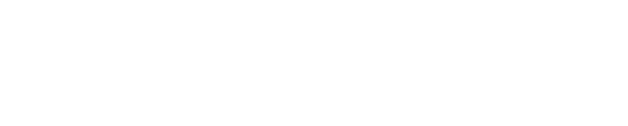

\author{
Allah Haafiz' \\ Christian Farrington' \\ Joel Andres' \\ Saleem Islam² \\ 'Hepatology and Liver \\ Transplantation, Division of Pediatric \\ Gastroenterology, Hepatology and \\ Nutrition, ${ }^{2}$ Division of Pediatric \\ Surgery, University of Florida College \\ of Medicine, Gainesville, FL, USA
}

\begin{abstract}
Background: Progressive hepatic fibrosis (HF) is a prominent feature of biliary atresia (BA), the most common indication for liver transplantation (LT) in children. Despite its importance in $\mathrm{BA}, \mathrm{HF}$ is not evaluated in routine patient care because the invasiveness of liver biopsy makes histologic monitoring of fibrosis unfeasible. Therefore, the identification of noninvasive markers to assess HF is desirable especially in children.
\end{abstract}

Purpose: The main goal of this pilot project was to establish an investigational framework correlating hepatic expression of fibrogenic markers with routine blood tests in BA.

Methods: Using liver explants from patients with BA $(n=26)$, immune-expression of connective tissue growth factor (CTGF), a key fibrogenic cytokine was determined using horseradishlabeled antibodies. Expression intensities of lobular (L-CTGF) and portal (P-CTGF) CTGF were determined by using ImageJ software. These CTGF intensities were correlated with blood tests performed at the time of LT. Correlation coefficients were determined for each blood test variable versus mean L-CTGF and P-CTGF expression intensities. A $P$-value of less than 0.05 was considered statistically significant.

Results: All patients had end-stage liver disease and persistent cholestasis at the time of LT. Kendall tau $(\tau)$ rank correlation coefficient for L-CTGF and white blood cell (WBC) was inversed $(-0.52$; $P \leq 0.02)$. Similar but statistically nonsignificant inverse relationships were noted between L-CTGF and prothrombin time (PT) $(-0.15 ; P \leq 0.4)$, international normalized ratio (INR) $(-0.14 ; P \leq 0.5)$, and platelet count $(-0.36 ; P \leq 0.09)$. Inversed $(\tau)$ rank correlation coefficients were also evident between P-CTGF expression and gamma-glutamyl transpeptidase (GGT), PT, INR, and platelet count. Pearson correlation coefficients for combinational analysis of standardized total bilirubin (TB), alkaline phosphatase, GGT, and platelet count with L-CTGF $(0.33 ; P=0.3)$ and P-CTGF (0.06; $P=0.8)$, were not significant. Similar analysis for alanine aminotransferase, TB, and GGT combination (L-CTGF, $0.16 ; P=0.5$; P-CTGF $-0.3 ; P=0.2$ ) as well as WBC, platelet count, and TB (L-CTGF: $-0.36 ; P=0.09$; P-CTGF $-0.33 ; P=0.13$ ) also revealed nonsignificant results.

Conclusion: Hepatic expression of fibrogenic markers can be correlated with routinely performed blood tests in patients with BA. We document that although a trend of inverse relationship is noted, hepatic CTGF expression does not correlate well with routinely performed blood tests in advanced BA. Further work is required to determine more reliable ways of noninvasive diagnosis of HF.

Keyword: connective tissue growth factor, liver fibrosis, blood tests, fibrogenesis

\section{Introduction}

Progressive hepatic fibrosis (HF) is a hallmark feature of biliary atresia (BA), the most common indication for liver transplantation (LT) in children. Although the exact

\footnotetext{
Correspondence: Allah Haafiz

University of Florida College of Medicine, Pediatric Gastroenterology, Hepatology, and Nutrition, PO Box 100296,

Gainesville, FL 326I0, USA

$\mathrm{Tel}+\mathrm{I} 3522739350$

Fax +I 3522739055

Email haafiab@peds.ufl.edu
}

submit your manuscript | www.dovepress.com

Dovepress

DOI: 10.2147/CEG.SI7145
Clinical and Experimental Gastroenterology 201 I:4 49-54

49

(c) 20I I Haafiz et al, publisher and licensee Dove Medical Press Ltd.This is an Open Access article which permits unrestricted noncommercial use, provided the original work is properly cited. 
pathogenesis of BA remains elusive, it is generally agreed that the BA-phenotype can result from diverse mechanisms of liver injury, provoking a stereotypic response comprised of inflammation, bile duct proliferation, apoptosis, and fibrogenesis. ${ }^{1}$ In this context, it is not surprising that HF is the most important predictor of the success of palliative Kasai porto-enterostomy (PE) surgery. ${ }^{2-4}$ However, assessment of $\mathrm{HF}$ has not yet been incorporated into routine post-PE patient care, mostly due to a lack of reliable noninvasive markers for assessing liver fibrosis. For the same reasons, even with advanced cirrhosis due to BA, the prognostic models utilized for listing patients for LT incorporate clinical and biochemical variables not related to the process of liver fibrosis. ${ }^{5,6}$ As such, identification and validation of noninvasive tools for assessing liver fibrosis can have a major favorable impact on post-PE patient care, especially desirable to overcome the drawbacks of liver biopsy. ${ }^{7,8}$ Although several putative serologic markers of fibrosis have been studied in adults such as hepatic aminotransferases and coagulation profiles, these tests can only partially reflect the extent of hepatic dysfunction, and they fail to reflect the balance between extracellular matrix (ECM) production and resolution. Similarly, assays of markers of ECM synthesis and degradation, despite their mechanistic appeal, only provide a cross-sectional glimpse of otherwise dynamic fibrogenic processes.

Connective tissue growth factor (CTGF), a 349-residue cysteine-rich protein first described as a secretory product of the vascular endothelial cell, ${ }^{9}$ is now the focus of intense research as a master fibrogenic cytokine involved in liver fibrosis due to a variety of liver diseases ${ }^{10-12}$ and other organs. ${ }^{13-23}$ Although only limited information is available in the literature about hepatic CTGF-expression in BA, ${ }^{24,25}$ it has been documented that not only that serum levels of CTGF were higher in patients with BA, but that these levels also correlated with worsening liver function. ${ }^{26}$ Liver fibrosis was not assessed in this study, thus leaving the question: can serum CTGF level be utilized as a marker of severity of HF in this disease? To answer this question, related and perhaps more fundamental questions requiring elaboration include: can hepatic-CTGF-expression be quantified at different stages of fibrosis, and if so, can the hepatic CTGF-expression (portal [P-CTGF] and lobular [L-CTGF]) be correlated with routinely performed blood tests? To address these questions and to establish a framework for the evaluation of CTGF as a biomarker of HF, our goal in this study was to determine whether routinely performed blood tests correlate with hepatic-CTGF-expression in advanced BA. To accomplish this goal, CTGF expression was determined by immunohistochemistry on the liver explant specimens obtained from children requiring LT for BA. The intensity of CTGF expression was then correlated with blood tests performed at the time of LT.

\section{Materials and methods Ethical considerations and access to human liver tissues and blood results}

After approval from the Institutional Review Board of the University of Florida (UF), BA patients requiring liver transplantation between 2000 and 2008 were identified from the UF liver transplant database $(n=26,14$ male, 12 female). The mean age at the time of liver transplantation was $1.8 \pm 0.369$ years (range, 2 months to 17 years). Blood levels of aspartate aminotransferase (AST), alanine aminotransferase (ALT), alkaline phosphatase (ALP), total bilirubin (TB), albumin, total protein (TP), prothrombin time (PT), international normalized ratio (INR), white blood cell (WBC) count, and platelet counts performed at the time of LT were recorded. Archived liver sections of living-related-liver-donors were used as controls. All biopsies were $4 \mu \mathrm{m}$ thick, fixed in phosphate-buffered formalin, and embedded in paraffin. At a randomly selected site of the explant, serial adjacent sections were used for hematoxylin and eosin staining immunohistochemistry, and trichrome staining. As we have recently reported, ${ }^{27}$ the stage of liver fibrosis was determined in a blinded fashion by using trichrome-stained slides according to the METAVIR scoring system.

\section{CTGF immunohistochemistry}

After rehydration, $4 \mu \mathrm{m}$ sections of paraffin-embedded tissue on glass slides were incubated in 3\% hydrogen peroxide to block the endogenous peroxidase activity followed by incubation in 3\% bovine serum albumin in phosphate buffered saline (PBS). A polyclonal CTGF antibody (Abcam ab6992) was applied at 1:1000, diluted in 3\% bovine serum albumin and incubated at $4{ }^{\circ} \mathrm{C}$ overnight. This antibody has been extensively used for immune localization of CTGF in variety of tissues. ${ }^{28-30}$ After being washed for 5 minutes in PBS, the slides were treated with goat anti-rabbit IgG horseradish peroxidase-labeled secondary antibodies (KPL, 474-1516) at a dilution of 2:500 for 30 minutes at room temperature. Bound antibodies were detected with an avidin-biotin complex (ABC) kit (Vector Laboratories, Burlingame, CA). The slides were stained with diaminobenzidine, washed, counterstained with Mayer's hematoxylin, dehydrated, treated with xylene, and mounted. 


\section{Determination of intensity of L-CTGF and P-CTGF expression}

The intensities of the L-CTGF and P-CTGF were determined using ImageJ software (http://rsb.info.nih.gov/ij). From each slide, five random portal and lobular areas were analyzed with a color histogram and a color deconvolution plug-in routinely used for diaminobenzidine staining. ${ }^{31}$

\section{Data analysis}

Our hypothesis was that the relationship between hepatic expression of CTGF and the routinely performed blood tests can be established. To test the hypothesis, correlation coefficients were calculated using SAS software (SAS Institute, Inc, Cary, NC). The response variables selected for the analysis were the mean intensities of L-CTGF and P-CTGF. The dependent variables included in this analysis were AST, ALT, ALP, gamma-glutamyl transpeptidase (GGT), TB, direct bilirubin (DB), TP, albumin, PT, INR, hemoglobin, hematocrit, WBC, and the platelet count. These variables were selected to represent cholestasis, a prominent feature of advanced $\mathrm{BA}$, and the common complications of BA including portal hypertension and chronic liver failure. For the corelational analysis, Kendall's tau correlation coefficients were determined for each dependent variable versus mean L-CTGF and P-CTGF expression intensities. A $P$-value of less than 0.05 was considered statistically significant. In addition, by way of principal component method, the coefficients for the linear combinations of standardized dependent variables were calculated. These combinations were determined to reflect typical pathophysiologic manifestations of BA.
For example, to assess the effect of the degree of cholestasis and portal hypertension, a linear-combination of TB, ALP, GGT, and platelet count were used to determine the Pearson correlation coefficient for L-CTGF and P-CTGF. Similar combinational analysis for ALT, TB, and GGT as well as WBC, platelet count, and TB were calculated. Because children with advanced BA frequently receive exogenous albumin and vitamin $\mathrm{K}$ supplements, to avoid a confounding effect, albumen and INR were not incorporated in any of the combinational analyses.

\section{Results}

All of the explant liver specimens exhibited advanced biliary cirrhosis, a typical sequel of BA requiring LT. Clinically, all patients had end-stage liver disease and persistent cholestasis (mean TB of $14 \mathrm{mg} / \mathrm{dL}$ ) at the time of LT. A summary of analysis of each of the dependent variables is provided in Table 1. Kendall's tau correlation coefficients for each dependent variable and the mean P-CTGF is shown in Table 2, whereas Table 3 outlines the correlation coefficients for each dependent variable and mean L-CTGF. As seen in Table 2, GGT, PT, INR, and platelet count had an inverse relationship with P-CTGF expression. In contrast, all other variables had a positive correlation with P-CTGF. However, as noted in the tables, it is important to recognize that most of the positive and reciprocal relationships are not statistically significant. Interestingly, as compared with P-CTGF, stronger inverse relationships were noted in the corelational analysis of L-CTGF. For example, as seen in Table 3, the correlation coefficient for L-CTGF and WBC was inversed

Table I Summary of dependent variables at the time of liver transplantation

\begin{tabular}{|c|c|c|c|c|}
\hline Variable & Mean \pm SEM & Median & Minimum & Maximum \\
\hline AST (U/L) & $264 \pm 39$ & 211 & 67 & 960 \\
\hline $\mathrm{ALT}(\mathrm{U} / \mathrm{L})$ & $181 \pm 35$ & 139 & 39 & 894 \\
\hline $\operatorname{ALP}(U / L)$ & $647 \pm 65$ & 665 & 81 & 1513 \\
\hline GGT (U/L) & $282 \pm 61$ & 209 & 15 & 825 \\
\hline TB (mg/dL) & $14 \pm 1.8$ & 13 & 1.4 & 34 \\
\hline Direct bilirubin (mg/dL) & $9 \pm 2$ & 7.6 & 0.1 & 23 \\
\hline Total protein (mg/dL) & $5.8 \pm 0.2$ & 5.8 & 2.2 & 7.3 \\
\hline Albumin (mg/dL) & $2.9 \pm 0.1$ & 3.1 & 1 & 3.9 \\
\hline Prothrombin time (seconds) & $16.8 \pm 0.5$ & 16.7 & 12.7 & 24.5 \\
\hline International normalized ratio & $1.5 \pm 0.05$ & 1.5 & 1.1 & 2.2 \\
\hline Hemoglobin $(g / d L)$ & $10 \pm 0.3$ & 10.2 & 7.2 & 12.9 \\
\hline Hematocrit $(29.0 \%-41.0 \%)$ & $31 \pm 0.8$ & 31.5 & 22.7 & 40 \\
\hline WBC $(6.0-17.5) \times 10^{9} / \mathrm{L}$ & $11 \pm 0.9$ & 1.3 & 1.7 & 20.5 \\
\hline Platelets $(150-450) \times 10^{9} / \mathrm{L}$ & $219 \pm 28$ & 214 & 54 & 780 \\
\hline
\end{tabular}

Notes: Results of the blood tests performed (Variable column) at the time of liver transplantation were retrieved from University of Florida transplant database. GraphPad was used to generate the summary of descriptive statistics.

Abbreviations: AST, aspartate aminotransferase; ALT, alanine aminotransferase; ALP, alkaline phosphatase; GGT, gamma-glutamyltransferase; SEM, standard error of the mean; TB, total bilirubin; WBC, white blood cell count. 
Table 2 Correlational analysis of routinely performed blood tests (dependent variables) and P-CTGF expression

\begin{tabular}{lll}
\hline Variable & $\begin{array}{l}\text { Kendall tau }(\tau) \text { rank } \\
\text { correlation coefficient } \\
\text { (P-CTGF) }\end{array}$ & P-value \\
\hline AST & 0.48 & \\
ALT & 0.36 & 0.0282 \\
ALP & 0.06 & 0.0998 \\
GGT & -0.33 & 0.7839 \\
Total bilirubin & 0.31 & 0.1797 \\
Direct bilirubin & 0.29 & 0.1682 \\
Total protein & 0.02 & 0.1916 \\
Albumin & 0 & 0.9452 \\
Prothrombin time & -0.15 & 1.0000 \\
International normalized ratio & -0.11 & 0.4908 \\
Hemoglobin & 0.11 & 0.6243 \\
Hematocrit & 0.22 & 0.6304 \\
WBC & -0.18 & 0.3348 \\
Platelets & -0.33 & 0.4106 \\
\hline Notes:Heptic CTGF expression & BA was & 0.1314 \\
\hline
\end{tabular}

Notes: Hepatic CTGF expression in BA was determined by immunostaining of liver-explants $(n=26)$ by using commercially available primary antibodies. Means of color pixel histograms representing the intensities of P-CTGF expression was determined by Image software. Kendall tau $(\tau)$ rank correlation coefficients (middle column) were then determined for the mean intensities of P-CTGF and blood tests performed at the time of LT (left-hand column).

Abbreviations: AST, aspartate aminotransferase; ALT, alanine aminotransferase; ALP, alkaline phosphatase; BA, biliary atresia; CTGF, connective tissue growth factor; GGT, gamma-glutamyltransferase; LT, liver transplantation; P-CTGF, portal CTGF; WBC, white blood cell count.

$(-0.52 ; P \leq 0.02)$. Similar inverse relationships were noted between L-CTGF and PT, INR, and platelet count $(-0.36$; $P \leq 0.09)$. Pearson correlation coefficients for combinational analysis of standardized TB, ALP, GGT, and platelet with L-CTGF $(0.33 ; P=0.3)$ and P-CTGF $(0.06 ; P=0.8)$, were not significant. Similar analysis for ALT, TB, and GGT combination (L-CTGF, $0.16 ; P=0.5$; P-CTGF $-0.3 ; P=0.2$ ) as well as WBC, platelet count, and TB (L-CTGF: -0.36 ; $P=0.09$; P-CTGF $-0.33 ; P=0.13)$ also revealed nonsignificant results. In summary, hepatic CTGF expression is noted to have an inverse albeit nonsignificant relationship with routinely performed hematological parameters. This is best depicted by L-CTGF expression and poorly by the P-CTGF expression. Both L-CTGF and P-CTGF had poor correlation with combinational analysis of dependent variables.

\section{Discussion}

Identification and validation of novel noninvasive surrogate markers of liver fibrosis has been slow due to poor elucidation of the mechanisms of fibrogenesis. This is especially true in pediatric cholestatic liver disorders such as BA, in which, a systematic approach to understanding the mechanisms of liver fibrogenesis has been lacking. Similarly, even in chronic liver disease affecting adults, various noninvasive methods to
Table 3 Correlational analysis of dependent variables and L-CTGF expression

\begin{tabular}{lll}
\hline $\begin{array}{l}\text { Dependent } \\
\text { variable }\end{array}$ & $\begin{array}{l}\text { Correlation coefficient } \\
\text { (L-CTGF) }\end{array}$ & P-value \\
\hline AST & 0.09 & 0.6 \\
ALT & 0.21 & 0.3 \\
ALP & 0.09 & 0.6 \\
GGT & 0.11 & 0.6 \\
Total bilirubin & 0.03 & 0.8 \\
Direct bilirubin & 0.05 & 0.8 \\
Total protein & 0.29 & 0.1 \\
Albumin & 0.13 & 0.5 \\
Prothrombin time & -0.15 & 0.4 \\
International normalized ratio & -0.14 & 0.5 \\
Hemoglobin & 0.38 & 0.08 \\
Hematocrit & 0.52 & 0.02 \\
WBC & -0.52 & 0.02 \\
Platelets & -0.36 & 0.09 \\
\hline
\end{tabular}

Notes: Immunostaining and hepatic L-CTGF expressions were determined as outlined in Table 2. Using SAS statistical software, Kendall tau $(\tau)$ rank correlation coefficients (middle column) were determined for the intensities of L-CTGF and blood tests performed at the time of liver transplantation (left-hand column).

Abbreviations: AST, aspartate aminotransferase; ALT, alanine aminotransferase; ALP, alkaline phosphatase; GGT, gamma-glutamyltransferase; L-CTGF, lobular connective tissue growth factor; WBC, white blood cell count.

quantify liver fibrosis are still in the process of evaluation and no marker, individually or as a composite index has replaced liver biopsy. For example, a systematic review of 14 studies of fibrosis biomarkers in patients with chronic hepatitis $\mathrm{C}$ (HCV) concluded that the panels of biomarkers could not differentiate stages of fibrosis accurately. ${ }^{32}$ Therefore, any putative biomarker of fibrosis such as CTGF requires careful investigations in a variety of liver diseases to determine its potential use as a biomarker of liver fibrosis. An ideal noninvasive fibrosis biomarker must be: liver specific; independent of metabolic alterations in liver, renal, or reticuloendothelial function; easy to perform; minimally altered by urinary or biliary excretion; reflective of fibrosis in all types of chronic liver injury; sensitive enough to discriminate between different stages of fibrosis; able to correlate dynamic changes in fibrosis progression or regression; and able to predict clinical outcomes, including liver failure and mortality. The discovery of novel biomarkers fulfilling all of these criteria is a challenge that will require concurrent understanding of the cellular process and the molecular mechanisms involved in hepatic fibrogenesis. At present, no single or panel marker fulfills all of these criteria sufficiently to merit routine clinical use.

In this pilot project, we document that the relationship between hepatic CTGF and routinely performed blood tests can be established by quantitative assessment of immunebased CTGF expression. Pertinent observations of our study include the following: 1) L-CTGF is better correlated 
with hematological parameters than P-CTGF; 2) an inverse relationship exists between WBC, PT, INR, and platelet count with L-CTGF expression in advanced BA; and 3) combination of dependent variables did not correlate with hepatic L-CTGF or P-CTGF expression. Interestingly, of all the dependent variables, WBC was noted to be most (inversely) corelated with L-CTGF-expression. This observation is important, given that WBC count is frequently depressed due to portal hypertension and splenomegaly in the setting of advanced cirrhosis in patients with BA. Apart from the inverse relationship between $\mathrm{WBC}$ and L-CTGF, most of the correctional coefficients were nonsignificant, suggesting that, even if CTGF plays a role in fibrogenesis, its expression does not correlate with characteristic hematological and biochemic abnormalities encountered in cirrhosis due to BA. This is not surprising in that although CTGF has been well-documented to be overexpressed in a variety of liver pathologies, ${ }^{12,33,34}$ specific mechanisms of CTGF overexpression remain obscure; thereby, its diagnostic or prognostic applications remain limited at the moment. Furthermore, apart from its fibrogenic potential, CTGF has an overarching biologic significance as a regulator of multiple cellular functions such as cell adhesion, mitogenesis, chemotaxis, proliferation, differentiation, neovascularization, apoptosis, and cell survival. ${ }^{35}$ Therefore, despite the compelling reasons to identify and validate novel biomarkers of HF, it appears quite reasonable to pause and ask: has the promise of CTGF to serve as a biomarker of HF been anticipated prematurely - without adequately elucidating underlying disease-specific mechanisms of its overexpression? Further data analyzing similar corelations from diverse liver diseases will help answer this question. However, in this study, one step proximal to its utilization as a biomarker of HF, hepatic CTGF expression is noted to be poorly correlated with routine blood tests and markers of progressive liver disease such as cholestasis, the cardinal feature of BA. However, several issues are quite important to recognize for the appropriate interpretation of the results presented in our study.

For example, due to the relatively small sample size, the scope of this report is restricted to mainly establishing a practical framework for the investigation of novel hepatic fibrogenic markers and their potential clinical use as biomarkers of liver fibrosis. Given that BA is a relatively uncommon disease, multicenter studies are needed to assess the reproducibility of this work with a larger sample size. Other pertinent caveats about this report include the uniform nature of advanced liver cirrhosis, and the absence of a comparative control group. Both issues have relevance to the presented results, and we recognize that these are best addressed by prospective multicenter collaborative studies. Therefore, rather than making conclusions against or in favor of CTGF as a biomarker of liver fibrosis, we suggest that this work is viewed as one step forward to framing pertinent questions and knowing how to answer these questions. For example, immediate questions emerging from this work include: does blood level of CTGF correlate with hepatic-CTGF-expression; and, if so, can blood CTGF levels be used as a surrogate for liver fibrosis. Due to ethical restriction on repeated biopsies, further work using experimental BA is needed to best address these questions. We are using rotavirus-induced BA to determine the usefulness of CTGF as a biomarker of HF in BA which may eventually encourage prospective multicenter investigations.

\section{Acknowledgment}

This work was supported by funds from the Children's Miracle Network. Publication of this article was funded in part by the University of Florida Open-Access Publishing Fund. We thank Marda Jorgensen for the technical advice on immunohistochemistry.

\section{Disclosure}

The authors report no conflicts of interest in this work.

\section{References}

1. Narkewicz MR. Biliary atresia: an update on our understanding of the disorder. Curr Opin Pediatr. 2001;13:435-440.

2. Davenport M, Howard ER. Macroscopic appearance at portoenterostomy - a prognostic variable in biliary atresia. $J$ Pediatr Surg. 1996;31:1387-1390

3. Shteyer E, Ramm GA, Xu C, White FV, Shepherd RW. Outcome after portoenterostomy in biliary atresia: pivotal role of degree of liver fibrosis and intensity of stellate cell activation. J Pediatr Gastroenterol Nutr. 2006;42:93-99.

4. Weerasooriya VS, White FV, Shepherd RW. Hepatic fibrosis and survival in biliary atresia. J Pediatr. 2004;144:123-125.

5. Jiang CB, Lee HC, Yeung CY, et al. A scoring system to predict the need for liver transplantation for biliary atresia after Kasai portoenterostomy. Eur J Pediatr. 2003;162:603-606.

6. Olthoff KM, Brown RS Jr, Delmonico FL, et al. Summary report of a national conference: Evolving concepts in liver allocation in the MELD and PELD era. December 8, 2003, Washington, DC, USA. Liver Transpl. 2004;10:A6-A22.

7. Abdi W, Millan JC, Mezey E. Sampling variability on percutaneous liver biopsy. Arch Intern Med. 1979;139:667-669.

8. Regev A, Berho M, Jeffers LJ, et al. Sampling error and intraobserver variation in liver biopsy in patients with chronic HCV infection. Am J Gastroenterol. 2002;97:2614-2618.

9. Bradham DM, Igarashi A, Potter RL, Grotendorst GR. Connective tissue growth factor: a cysteine-rich mitogen secreted by human vascular endothelial cells is related to the SRC-induced immediate early gene product CEF-10. J Cell Biol. 1991;114:1285-1294.

10. Kanzler S, Baumann M, Schirmacher P, et al. Prediction of progressive liver fibrosis in hepatitis $\mathrm{C}$ infection by serum and tissue levels of transforming growth factor-beta. J Viral Hepat. 2001;8:430-437. 
11. Nelson DR, Gonzalez-Peralta RP, Qian K, et al. Transforming growth factor-beta 1 in chronic hepatitis C. J Viral Hepat. 1997;4:29-35.

12. Williams EJ, Gaca MD, Brigstock DR, Arthur MJ, Benyon RC. Increased expression of connective tissue growth factor in fibrotic human liver and in activated hepatic stellate cells. J Hepatol. 2000;32:754-761.

13. Cimica V, Batusic D, Haralanova-Ilieva B, et al. Serial analysis of gene expression (SAGE) in rat liver regeneration. Biochem Biophys Res Commun. 2007;360:545-552.

14. George J, Tsutsumi M. siRNA-mediated knockdown of connective tissue growth factor prevents $\mathrm{N}$-nitrosodimethylamine-induced hepatic fibrosis in rats. Gene Ther. 2007;14:790-803.

15. Gressner OA, Weiskirchen R, Gressner AM. Evolving concepts of liver fibrogenesis provide new diagnostic and therapeutic options. Comp Hepatol. 2007;6:7-1.

16. Grotendorst GR, Okochi H, Hayashi N. A novel transforming growth factor beta response element controls the expression of the connective tissue growth factor gene. Cell Growth Differ. 1996;7:469-480.

17. Grotendorst GR. Connective tissue growth factor: a mediator of TGF-beta action on fibroblasts. Cytokine Growth Factor Rev. 1997;8: 171-179.

18. Igarashi A, Nashiro K, Kikuchi K, et al. Connective tissue growth factor gene expression in tissue sections from localized scleroderma, keloid, and other fibrotic skin disorders. J Invest Dermatol. 1996;106: 729-733.

19. Igarashi A, Okochi H, Bradham DM, Grotendorst GR. Regulation of connective tissue growth factor gene expression in human skin fibroblasts and during wound repair. Mol Biol Cell. 1993;4:637-645.

20. Ito Y, Aten J, Bende RJ, et al. Expression of connective tissue growth factor in human renal fibrosis. Kidney Int. 1998;53:853-861.

21. Morikawa H, Tamori A, Nishiguchi S, et al. Expression of connective tissue growth factor in the human liver with idiopathic portal hypertension. Mol Med. 2007;13:240-245.

22. Narkewicz MR, Kasaragod A, Lucia MS, Pflummer S, Sokol RJ, Stenmark KR. Connective tissue growth factor expression is increased in biliary epithelial cells in biliary atresia. J Pediatr Surg. 2005;40: $1721-1725$.

23. Oemar BS, Luscher TF. Connective tissue growth factor: friend or foe? Arterioscler Thromb Vasc Biol. 1997;17:1483-1489.
24. Narkewicz MR, Kasaragod A, Lucia MS, Pflummer S, Sokol RJ, Stenmark KR. Connective tissue growth factor expression is increased in biliary epithelial cells in biliary atresia. J Pediatr Surg. 2005;40: $1721-1725$.

25. Kobayashi H, Puri P, O’Briain DS, Surana R, Miyano T. Hepatic overexpression of MHC class II antigens and macrophage-associated antigens (CD68) in patients with biliary atresia of poor prognosis. J Pediatr Surg. 1997;32:590-593.

26. Tamatani T, Kobayashi H, Tezuka K, et al. Establishment of the enzyme-linked immunosorbent assay for connective tissue growth factor (CTGF) and its detection in the sera of biliary atresia. Biochem Biophys Res Commun. 1998;251:748-752.

27. Farrington C, Novak D, Liu C, Haafiz AB. Immunohistochemical localization of transforming growth factor $\beta-1$ and its relationship with collagen expression in advanced liver fibrosis due to biliary atresia. Clin Exp Gastroenterol. 2010;3:185-191.

28. Yang L, Besschetnova TY, Brooks CR, Shah JV, Bonventre JV. Epithelial cell cycle arrest in G2/M mediates kidney fibrosis after injury. Nat Med. 2010;16:535-543.

29. Qiu Z, Cang Y, Goff SP. c-Abl tyrosine kinase regulates cardiac growth and development. Proc Natl Acad Sci U S A. 2010;107:1136-1141.

30. Liu S, Taghavi R, Leask A. Connective tissue growth factor is induced in bleomycin-induced skin scleroderma. J Cell Commun Signal. 2010;4: 25-30.

31. Grewal D, Jain R, Brar GS, Grewal SP. Pentacam tomograms: a novel method for quantification of posterior capsule opacification. Invest Ophthalmol Vis Sci. 2008;49:2004-2008.

32. Parkes J, Guha IN, Roderick P, Rosenberg W. Performance of serum marker panels for liver fibrosis in chronic hepatitis C. J Hepatol. 2006;44:462-474.

33. Paradis V, Dargere D, Vidaud M, et al. Expression of connective tissue growth factor in experimental rat and human liver fibrosis. Hepatology. 1999;30:968-976.

34. Rachfal AW, Brigstock DR. Connective tissue growth factor (CTGF/CCN2) in hepatic fibrosis. Hepatol Res. 2003;26:1-9.

35. Moussad EE, Brigstock DR. Connective tissue growth factor: what's in a name? Mol Genet Metab. 2000;71:276-292.
Clinical and Experimental Gastroenterology

\section{Publish your work in this journal}

Clinical and Experimental Gastroenterology is an international, peerreviewed, open access journal, publishing all aspects of gastroenterology in the clinic and laboratory, including: Pathology, pathophysiology of gastrointestinal disease; Investigation and treatment of gastointestinal disease; Pharmacology of drugs used in the alimentary tract;

\section{Dovepress}

Immunology/genetics/genomics related to gastrointestinal disease. This journal is indexed on CAS. The manuscript management system is completely online and includes a very quick and fair peer-review system. Visit http://www.dovepress.com/testimonials.php to read real quotes from published authors. 